\title{
ABOUT THE SOIL LINE CONCEPT IN REMOTE SENSING
}

\author{
F. Baret, S. Jacquemoud and J. F. Hanocq \\ INRA Bioclimatologie, BP 91, 84143 Montfavet Cedex, France
}

\begin{abstract}
The soil line, a linear relationship between bare soil reflectance observed in two different wavebands, is widely used for interpretation of remotely sensed data. The basis on soil line was analyzed using a radiative transfer model in which reflectance was splitted into its single and multiple scattering components. The slope of the soil line corresponded to the ratio of the single scattering albedos corresponding to the two wavebands where the soil line was defined. The intercept originated from the difference in multiple scattering observed in each of the two wavelength bands used. The soil line concept was very robust over the whole optical domain as soon as soil types are separated, and when the effect of the view and source configurations as well as the surface roughness were considered. However, in the middle infrared spectral domain, the soil line concept failed when soil moisture was a factor of variation.
\end{abstract}

\section{INTRODUCTION}

Soil optical properties influence the radiometric response of canopies since the soil is the last bottom background. Soil reflectance is likely to change from place to place, depending on soil type. It may also change with the observation date for a specific location, depending on its surface status characterized by roughness and moisture, and eventually on the amount of vegetation residuals lcft on it. Therefore, radiometric data collected over vegetation have to be corrected to retain most of the information on canopies. Several indices were developed to minimize soil background influence. They generally combine reflectance measured in a few wavelength bands $/ 1,2 /$. Most of these so called vegetation indices are based on the assumption that bare soil reflectance lies on a single line in the space generated by the wavelength bands. This line is termed as soil line. Vegetation indices are often a measure of the departure from this line, either using the Euclidean distance or the angular difference $13,4 /$. A soil line results from the combined variations of its roughness, moisture and view or source configuration, for a given soil type and a set of wavelengths. Authors generally assume that a unique "global" soil line represents all soil types. However, studies. $15 /$ pointed out that specific soil lines described better the optical properties of individual soil types.

In this work, the basis of the soil line concept was investigated using a soil reflectance model. The possible factors of variations of the soil line were analyzed through a laboratory experiment where both spectral and directional reflectance variations of few contrasted soil types and surface aspects were measured.

\section{MODELING THE SOIL LINE}

A version of Hapke's model /6/ was successfully tested to describe the spectral and directional variations of soil reflectance $77,8 \%$. This model assumes that the reflectance $\rho$ is the sum of a single scattering $\rho_{\mathrm{s}}$ and a multiple scattering $\rho_{\mathrm{m}}$ components. Single scattering is described (equation (1)) by the single scattering albedo, $\omega$, a phase function, $P(g, g)$, and a function, $B(g, h)$ representing the backscattering depending on a roughness parameter $h . g$ and $g^{\prime}$ are the

$$
\begin{aligned}
& \rho_{m}=\frac{\omega}{4\left(\mu_{i}+\mu_{o}\right)}\left(H\left(\omega, \mu_{i}\right) H\left(\omega, \mu_{o}\right)-1\right) \\
& \rho_{s}=\frac{\omega}{4\left(\mu_{i}+\mu_{o}\right)}(1+B(g, h)) P(g)
\end{aligned}
$$
phase and anti phase (angle between the specular and the view directions) angles, $\mu_{\mathrm{i}}$ and $\mu_{0}$ are, respectively, the cosine of the incident and observation zenith angles. Multiple scattering (eqution (9)) was evaluated using Chandrasekhar /9/ function, $\mathrm{H}(\omega, \mu)$, assuming isotropic scattering. Input parameters of the model are the incidence and observation geometry, the roughness parameter $h$, the single scattering albedo $\omega$, and the 4 coefficients of the phase function $P\left(g, g^{\prime}\right)$ described by a modified Legendre polynomial. A complete description of the model was provided by Jacquemoud et al. /8/. These authors demonstrated that the 

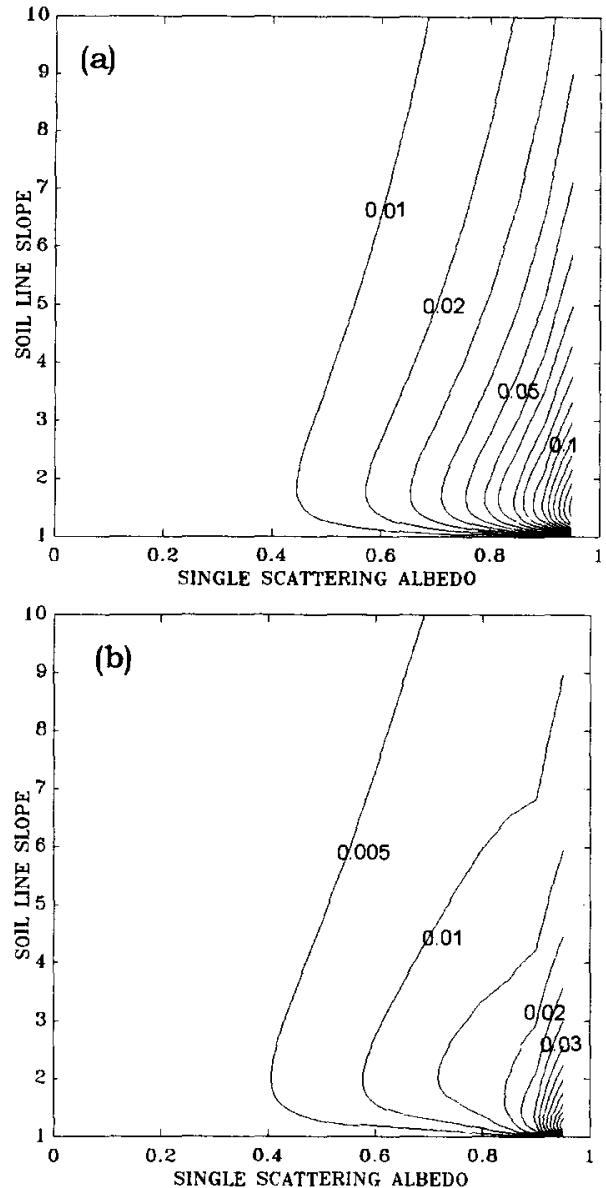

Fig. 1. Isocontour lines of the difference between the maximum and minimum values (a) and the average values (b) of the soil line intercept $\beta$ observed for given $\omega_{2}$ and $\alpha$ when the configuration varies $\left(\mu_{\mathrm{i}}\right.$ and $\mu_{0}$ vary from 0 to 1$)$. $\beta$ is computed using equation (4).

While in the theoretical study the fundamental of the soil line were clearly demonstrated from the variation of the model parameters, factors such as roughness, moisture or soil type were only implicitly related to the model parameters. The factors of variations of model parameters were subsequently identified using a laboratory experiment that will also provide direct evidence of the robustness of the soil line concept.

\section{EXPERIMENTAL EVIDENCE OF SOIL LINES.}

\section{The Experiment}

To fully control the experimental conditions, the study was conducted indoor. A Barringer Hand Held Ratioing Radiometer (HHRR) was mounted at the end of a $1.5 \mathrm{~m}$ pole rotating in a vertical plane, allowing the view zenith angle to vary. Five broad band filters permit to measure reflectance in the visible (538 and $631 \mathrm{~nm})$, near infrared $(85 \mathrm{lnm})$ and middle infrared $(1768$ and $2209 \mathrm{~nm})$. Soil samples were arranged in $0.25 \mathrm{~m}^{2}$ square boxes, placed horizontally at the same level as the pole axis. The rectangular field of view provided a sufficient spatial sampling in the direction perpendicular to the view axis $\left(15.6^{\circ}\right)$, while the narrow aperture $\left(2.6^{\circ}\right)$ along the rotation view axis permitted a fine description of the bi-directional reflectance features. The samples were illuminated alternatively by five $2000 \mathrm{~W}$ halogen lamps with quasi collimated light beams. One at nadir, 2 in the principal plane at $34^{\circ}$ and $60^{\circ}$ zenith angles and 2 in the perpendicular plane at the same zenith angles. For each soil sample, 42 view and source configurations were acquired in about 20 minutes. The signal output from the radiometer was converted into absolute bidirectional reflectance using a halon reference panel. A standard deviation close to 0.01 indicated a good measurement accuracy. To get large variations of soil optical properties, 26 contrasting soil types and 
surface conditions were sampled. They ranged from fine silica sand, peat and clayey soils, to pozzolona and pebbles.

\section{Effects of Various Factors on the Soil Line}

Model parameters were fitted for each of the 26 soils, assuming that the single scattering albedo was the only parameter spectrally dependent (Jacquemoud et al. $/ 8 /$ ). Non linear fitting techniques minimizing the Root Mean Square Error (RMSE) were used. The small RMSE (RMSE $=0.015, R^{2}=0.995$ ), computed over the whole data set ( 26 soils $\times 42$ configurations $\times 5$ wavelength bands $=5460$ data) indicates a very good match.

Soil line parameters were evaluated for each of the 26 samples and the 20 possible combinations of the 5 wavebands with measurement configuration as unique factor of variation of reflectance. RMSE were computed (Table 1) for all channels $(538,631,851,1768$ and $2209 \mathrm{~nm})$, for visible and near infrared only $(538,631$ and $851 \mathrm{~nm})$, or for the middle infrared only $(1768$ and $2209 \mathrm{~nm})$ ). Each time, distinction was made between cases with low to moderate values of single scattering albedo $(\omega<0.7)$ and all cases $(\omega<1.0)$.

When soil lines are evaluated for each soil type, roughness and moisture levels, the associated RMSE were very small, with an overall value of 0.013 (see Table 1, combination \#1). This confirmed the former theoretical findings. Further, the estimated slopes were in good agreement with the computed ratio of the single scattering albedos, as stated by equation 4 .

A variation of surface roughness for a given soil type at a fixed moisture level affected both the roughness parameter and the phase function. Single scattering albedo increased generally when the soil gets smoother because the aggregates were partly destroyed and the microscopic aspect of the surface changed. Single scattering albedo values observed in any two wavelengths did not exhibit clear linear behavior when soil roughness changes. Nevertheless, no significant scattering appears around soil lines when the various roughness levels were pooled together for given soil type and moisture level (Combination \#2 as compared to combination \#1 in Table 1). Further, this property seemed not to be wavelength dependent nor to be attenuated for high single scattering albedo values $(\omega>0.7)$.

Among model parameters, the single scattering albedo was the most sensitive to soil moisture $(\theta)$ changes. In spectral domains where water did not significantly absorb light, single scattering albedo of wet soil $\omega(\theta)$ was almost linearly related to that of the dry soil, $\omega\left(\theta_{0}\right)$. In visible and near infrared characterized by very small water absorption features, single scattering albedos varied in between the 1:1 line corresponding to dry soils, and the Angstrom's $/ 10 /$ curve approximating saturated soils (Figure 3). Because of the proximity of the first bisectrice and Angstrom's

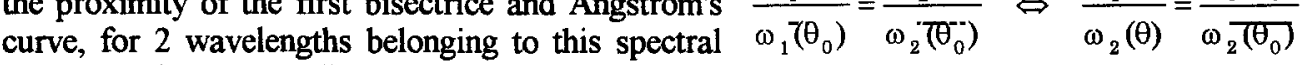
domain and corresponding to $w_{1}$ and $w_{2}$ we approximated by equation (5). Henceforth, according to the former theoretical findings, soil lines were expected in the visible and near infrared domain when soil moisture varied. The RMSE values (Table 1, Combination \#3) evaluated for each soil type when moisture levels were pooled together did not increase significantly in this spectral domain as compared to the previous situation (Combinations \#1). Conversely, in the middle infrared domain where water absorbed strongly, equation (5) did not apply (Figure 2) and no consistent soil line was expected, especially for bright

TABLE 1: Residual errors (RMSE) values of soil lines computed for several combinations of the factors of variation: Soil type, moisture and roughness levels.

\begin{tabular}{cccccccccc}
\hline \multirow{2}{*}{$\begin{array}{c}\text { Comb } \\
\text { in. \# }\end{array}$} & \multicolumn{2}{c}{ Factors of Var. Consid. } & \multicolumn{2}{c}{ AllChannels } & \multicolumn{2}{c}{ Vis. \& NIR } & \multicolumn{2}{c}{ Mid. IR. } \\
\cline { 2 - 10 } & Type & Moist. & Roug. & $\omega<1.0$ & $\omega<0.7$ & $\omega<1.0$ & $\omega<0.7$ & $\omega<1.0$ & $\omega<0.7$ \\
\hline 1 & $\times$ & $\times$ & $\times$ & 0.013 & 0.009 & 0.008 & 0.007 & 0.012 & 0.009 \\
2 & $\times$ & $\times$ & & 0.016 & 0.011 & 0.011 & 0.008 & 0.014 & 0.011 \\
$3^{*}$ & $\times$ & & $\times$ & 0.037 & $0.017^{* *}$ & 0.011 & 0.010 & 0.022 & 0.019 \\
4 & $\times$ & & & 0.041 & 0.021 & 0.013 & 0.011 & 0.024 & 0.022 \\
5 & & & & 0.091 & 0.077 & 0.038 & 0.044 & 0.045 & 0.035 \\
\hline \multicolumn{3}{r}{ Number. of Data Used } & 21840 & 12768 & 6552 & 4284 & 2184 & 1176 \\
\hline
\end{tabular}

\footnotetext{
Median soil moisture levels were not included because moisture mat have changed during the experiment from one roughness level to an other.

This number corresponds to a 0.017 RMSE value of soil lines computed over the 5 channels, for $\omega$ below 0.70 , when a distinction is made between the soil types and the roughness levels (moisture levels are pooled together for each soil type and roughness levels). To allow direct comparisons between various combinations of the factors of variation (vertically in the table), the RMSE values are evaluated over the same population (presented in the last line of the table) for each set of channels and $\omega$ threshold levels.
} 
soils. The increase of the RMSE values of combination \#3 observed in this spectral domain as compared to that of combination \#1 (Table 1) confirmed this result.

Each soil type corresponded to a particular set of soil line parameters. The scatter around soil lines measured by the RMSE was divided by 2 to 4 when the soil type was used as a factor of variation (compare combination \#4 and \#5 in table. 1). Soil type was obviously a major factor of variation of soil lines. The concept of a 'global' soil line should, thus, be definitively buried out.

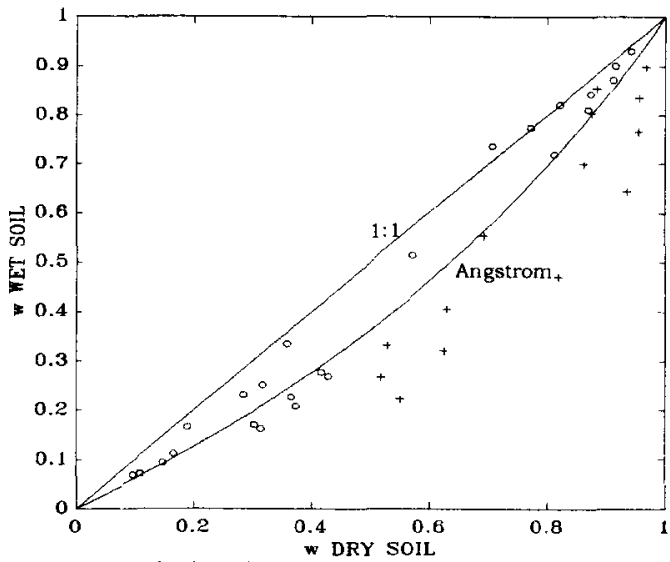

Fig. 2. Relationship between Dry and wet soil single scattering albedos. The ' $O$ ' and ' + ' correspond respectively to the visible-near infrared domain and middle infrared spectral domains. concept of a "global" soil line did not apply. The soil type appeared to be the main factor of variation of soil line parameters. However, results indicated that the soil line concept resisted to changes in surface roughness for a given soil type. This was still true for changes of surface moisture when observed over a given soil in the visible and near infrared spectral domains. Conversely, in the middle infrared spectral domain characterized by strong water absorption features, changes in surface moisture violated the soil line concept.

Acknowledgments: This study was supported by the French Programme National de Teledetection Spatiale with a contribution from CNES, CNRS, INRA and IFRFMER. Special thanks must be given to $\mathrm{B}$. Andrieu (INRA Grignon) who kindly borrowed us the HHRR Barringer radiometer, to C. Sarrouy and J. Saumade (INRA Montfavet) for their help and quiet comprehension of the "invasion" of the workshop. Many thanks are also due to B. Seguin (INRA Avignon) who presented this paper at the very end of the COSPAR meeting

\section{REFERENCES:}

1. R.J. Kauth and J.S. Thomas, The tasseled cap-a graphic description of the spectral-temporal development of agricultural crops as seen by Landsat, in: Machine processing of remotely sensed data. Purdue University, West Lafayette, Indiana, USA. 1976. p. 51.

2. A.J. Richardson and C.L. Wiegand, Distinguishing vegetation from soil background information. Photogramm. Eng. Remote Sens. 43, 1552 (1977).

3. A.R. Huete, Soil influences in remotely sensed vegetation-canopy spectra, in: Theory and application of optical remote sensing (G. Asrar ed.), Wiley interscience. 1989, p. 141

4. F. Baret and G. Guyot, Potentials and limits of vegetation indices for LAI and APAR assessment, Remote Sens. Environ. 35,161 (1991).

5. A.R. Huete, D.F. Post, and R.D. Jackson, Soil spectral effects on 4-space vegetation discrimination, Remote Sens. Environ. 15, 155 (1984).

6. B. Hapke, Bi-directional reflectance spectroscopy. 1. Theory, J. Geophys. Res. 86, 4571 (1981).

7. B. Pinty, M.M. Verstraete, and R.E. Dickinson, A physical model for predicting bi-directional reflectances over bare soils, Remote Sens. Environ. 27, 272 (1989).

8. S. Jacquemoud, F. Baret and J.F. Hanocq, Modelling spectral and directional soil reflectance, Remote Sens. Environ. 41, 123 (1992).

9. S. Chandrasekhar, Radiative transfer, Dover Publication, 1960.

10. A. Angstrom, The albedo of various surfaces of ground, Geografiska Ann. 7, 323.(1925) 